\title{
Photometric detection of pulsations in the stars of the wide binary WDS 12483-6708
}

\author{
D. Sinachopoulos, P. Gavras \\ Institute of Astronomy and Astrophysics, National Observatory of Athens \\ I. Metaxa and Bas. Pavlou, GR-15236 Athens, Greece
}

\begin{abstract}
We detected stellar pulsations in at least one of the components of the wide double star WDS 12483-6708. The largest observed photometric amplitude, in Stroemgren filter $y$, is around 0.02 magnitudes with period about 0.02 days. The detection was based on five hours of differential CCD photometry of the two double star components.
\end{abstract}

Individual Objects: WDS 12483-6708, HIC 62482, HIC 62488

\section{Introduction and Observations}

WDS $12483-6708$ is a wide double star detected in 1835. Since then, the relative positions of its components have been observed 16 times. The last observation listed in the Washington Double Star Catalogue (WDS) (Mason \& Hartkopf 2006) is from the year 2000, when the angular separation of the components measured was 13.5 arcseconds. The primary of the system is a A3III/IV type star. It is the HIC 62482 target of Hipparcos with $\mathrm{V}=7.58,(\mathrm{~B}-\mathrm{V})=0.19$ and parallax 10 mas. The secondary star is HIC 62488 with magnitude $V=8.74,(B-V)=0.47$ and has practically the same parallax of the primary. The proper motions of the two components are the same (common proper motion stars). These two facts show that this double star is not an optical pair. Hipparcos catalogue (ESA 1997) flags both components for 'duplicity-induced' variability.

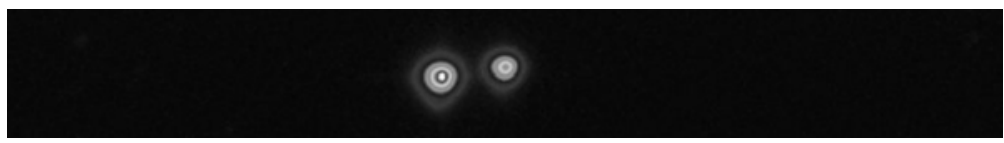

Figure 1: A characteristic CCD exposure of WDS 12483-6708 in Stroemgren $y$ filter 


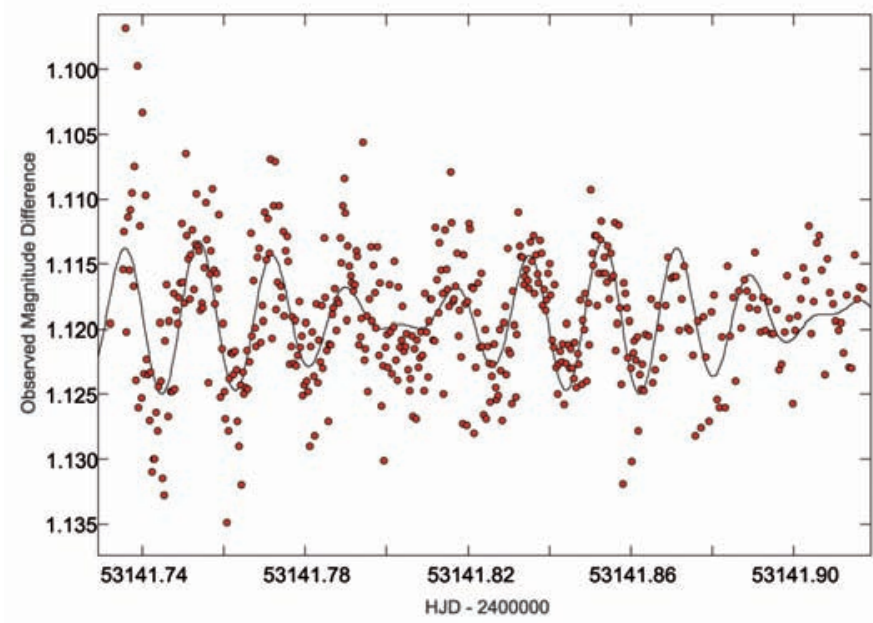

Figure 2: Variability of the magnitude difference of the components of WDS $12483-6708$, together with the fit of the two detected frequencies.

We observed this double star in May 16, 2004, in the frame of astrometric observations of wide Hipparcos visual double stars (Sinachopoulos et al. 2007), using the STE4 CCD camera of SAAO observatory attached to the Cassegrain focus of the $1 \mathrm{~m}$ telescope, using the Stroemgren $y$ filter. To improve the instrumental accuracy of our measurements, especially the astrometric ones, we obtained sixteen CCD exposures of each double star similar to the image shown in Figure 1.

Our -quasi- on-line data reduction resulted in an unexpectedly high value of the standard deviation of measured mean magnitude difference of the double star components. Since these two stars are in or close to the instability strip of the MS, we decided to spend five hours monitoring them photometrically. To our regret, there is no other star close WDS 12483-6708 bright enough which could be used as a comparison star, so we could only measure the variability of the magnitude difference of the two components. We took a series of ten-second exposures, which together with the CCD readout time resulted in 95 magnitude differences per hour. Our measurements are shown in Figure 2.

\section{Frequency analysis}

The light curve shows significant variability during the observational window. The largest amplitude we see in our measurements is about 0.02 mag. Consequently, Fourier analysis was performed using Period04 (Lenz \& Breger 2005). Two frequencies were detected and listed in Table 1 together with their semi-amplitudes and phases. Uncertainties are calculated by least-square fitting. Frequencies and phases 
Table 1: Observed frequencies for WDS 12483-6708 y-Data

\begin{tabular}{cccc}
\hline Id. & $\begin{array}{c}\text { Frequency } \\
{[\mathrm{c} / \mathrm{d}]}\end{array}$ & $\begin{array}{c}\text { Semi-ampl. } \\
{[\mathrm{mag}]}\end{array}$ & $\begin{array}{c}\text { Phase } \\
{[\text { cycles }]}\end{array}$ \\
\hline$f_{1}$ & $50.6 \pm 0.3$ & $0.0032 \pm 0.0003$ & $0.52 \pm 0.01$ \\
$f_{2}$ & $60.1 \pm 0.3$ & $0.0027 \pm 0.0003$ & $0.54 \pm 0.02$ \\
\hline
\end{tabular}

are fitted uncorrelated. The difference of $9.5 \mathrm{c} / \mathrm{d}$ between the two detected frequencies is significantly larger than the proposed frequency resolution of $1.5 / \mathrm{dT}$ limit by Loumos \& Deeming (1978), which in this case is $7.2 \mathrm{c} / \mathrm{d}$. Residuals to the observations are 0.004 magnitudes.

\section{Discussion and Conclusion}

Despite the relatively low level of the photometric residuals, the two frequencies fitted to the data and shown in Figure 2 give rise to the need for additional observations. These should include observations of external comparison and check stars to uncover the variable component of the wide, without excluding the scenario of finding both of them pulsating.

In any case, we were not able to locate any variable star previously known at these coordinates in the sky. This object is not listed in the Delta Scuti catalogue by Rodriguez et al. (2000). So, this paper reports the detection of at least one additional pulsating star in a wide double star system.

Acknowledgments. We would like to thank SAAO for generous telescope time allocation.

\section{References}

Perryman, M. A. C., \& the Hipparcos Science Team 1997, The Hipparcos and Tycho Catalogues (ESA SP-1200; Noordwijk: ESA)

Lenz, P., \& Breger, M. 2005, CoAst 146, 53

Loumos, G.L., \& Deeming, T.J. 1978, ApSS 56, 285

Mason, B.D., \& Hartkopf, W.I. 2006, JDSO 2, 171

Rodriguez, E., Lopez-Gonzalez, M. J., \& Lopez de Coca, P. 2000, A\&AS 144, 469

Sinachopoulos, D., Gavras, P., Dionatos O., et al. 2007, A\&A 472, 1055 